\title{
Study on the Usability of household electrical appliances for the aged
}

\author{
Huiping Liang \\ Guilin University of Electronic and Technology, Guilin, China \\ 20341863@qq.com
}

\section{Keywords: Household electrical appliances; Usability; the aged; Multi function}

Abstract: This article focus on the human aging population, the potential demand for elderly people pay attention to the development of times, the home appliance products as the research background, the elderly as the research object, mining potential is more suitable for old people to use products and the elderly market. Methods to analyze and understand the psychological and physiological characteristics of the elderly in the age of the age of social aging in our country. Research and design on the main point of the use of easy to use household electrical appliances. On the basis of the above, the expectation and design elements of the aged people in the use of the product were studied by the example of the rice cooker. Conclusion for the elderly to expect the demand for household electrical appliances to further design and research, through the design, to the elderly a healthy, safe and convenient household electrical appliances.

\section{Introduction}

With the development of economy, people's living standards improve, people's life expectancy increases, the aging of the world is more and more obvious. The aging process of our country has entered a stage of rapid development. As of the end of 60, China's elderly population aged over 190 million is about 2012, accounting for 14\% of the total population. In 2013 China Committee on Aging office announced that in 2013 the elderly population over the age of 60 will exceed 200 million in the next 20 years the average annual growth of about 10 million elderly people, and by 2050, the elderly population will reach $1 / 3$ of the country's population is still increasing every year at the speed of 8 million. Therefore, the aging industry has also become the potential of the "sunrise industry" in twenty-first Century, the development and development of products to meet the needs of the elderly more and more products become a trend.

\section{User Research and needs analysis of the elderly Section Headings.}

\section{Elderly users study}

The domestic and foreign definitions for the elderly are different, the developed countries will be 65 years old and above person for the elderly in China is defined at the age of 60 and above for the elderly, the average life expectancy of the global human and physical qualities of the United Nations WHO were determined and defined elderly age: young and old young people (60 74 years old), the elderly, their health status is good, and there is a certain consumption ability and self-care ability. Old people (75 years old $\sim 89$ years old), the age of the elderly due to various aspects of the body's functions are beginning to decline, whether mental or physical are not as big as before. At this stage the most need to exchange and care of their children, the need for close relatives of the psychological care of the patient. Elderly people (aged 90 and above), the health of the elderly in this age group is declining, the independence of the lower, so more emphasis on conservation. 


\section{Physiological function of the elderly}

People to the elderly, the physiological characteristics of the change is very obvious, all kinds of organs will be aging, the function of the organ will change. Mainly in the following aspects:

First, the decline in sensory function, the elderly with the age of growth, the body parts of the performance of different degrees of decline, mainly manifested as: vision, hearing loss, the response to things obviously more slow. Directly affect the elderly to use the rice cooker, in the button, control, material aspects to give consideration.

Second, motor function decline, with the growth of age, the elderly body's various organs, tissues and other functions appear obvious decline. At the same time, blood pressure and exercise organs and so on also with the growth of age and a series of degradation, such as muscle atrophy, decreased flexibility, coordination, etc.. In the design of the elderly, the motor function of the elderly should be considered.

Third, psychological function decline into old age, changed the old range of activities is relatively free, the kitchen has become another of their positions, they need emotional communication, self realization, valued and respected, which reflect their own values. And with age, the growth of memory loss, especially after the age of 60 decreased significantly.

Step into the old age, they begin to have the characteristics of the elderly population. In the design process, we should consider the elderly sensory function, motor function, psychological problems, such as vision, memory decline and other problems we have in the design of rice pot, to solve the elderly to use.

\section{Emotional analysis of the elderly}

With the change of age, the age of the elderly, the changes in the environment, emotion also showed different performance in the past. Mainly in the following aspects:

First, a sense of security, as the elderly population aging in terms of physical function, the lack of a sense of security, the complexity and difficulty of the operation, fear of failure prone to danger, to cause adverse effects on physical and mental health. Therefore, in the use of kitchen rice cooker, safety is also one of the basic psychological needs of the elderly group.Second, the sense of autonomy, older age groups on a certain age, the psychological slowly become fragile, relatively easy to sensitive, eager to get the family, friends, respect and love. Older people prefer to be independent and do what they want to do. For a certain complexity of the operation of the product, it is easy to cause their emotional pessimism, and for the elderly kitchen appliances operating convenience, it can enhance the confidence of the elderly and operational autonomy.

\section{Cognitive and consumer psychology of the elderly}

As the age increases, the cognitive ability of the old people is declining, which is mainly reflected in the aspects of memory, attention and perception. Their ability to control the use of rice in the pot has been reduced, the ability to read, analyze and decision to display information is also reduced.

Due to different ways of life of the elderly, consumption concept is also different from other age groups, summed up as two points:

The first reason, the old people in the consumer concept, can maintain an objective way to buy, to the real needs of the guide.

Second practical, convenient, safe, older people pay more attention to the practical functions of the product, pay attention to whether the product is to solve the problem, whether it is easy to use, whether it is safe or not. Compared to cool appearance, older people are more like natural, simple. Thus, the focus of the design of the rice cooker is put forward, one is convenient to use, and the operation is simple. Two, the function is simple, economical and durable, in the design process of the rice cooker, and strive to achieve these two points, to cater to the old people's consumption psychology. Therefore, the cognitive characteristics of the elderly determines the reliability and safety of the elderly kitchen cooker design. 
Use the habit of analysis

After exiting the mainstream, the elderly population has reduced social participation. Although much more free time than before, but the physical changes, the elderly to the kitchen and control, not so handy, plus psychological become more sensitive, the degree of concern for their safety is higher, but also the fear of some products. The use of strange products for the elderly, will be based on past life experience to solve the problem. For example, the older people are more accustomed to using the knob. For the use of electronic products, they are more accustomed to using large screen control.

\section{Analysis of the ease of use of the product in the elderly}

Due to the old people's ability to reflect the decline has begun to decline, the ability to accept new things is relatively weak, therefore, suitable for the elderly to have a simple and easy to learn the characteristics of the product. At the same time for the old people, the security design is an important aspect for falling reaction ability of old people, should be more active defense design on the safety design, in order to protect the safety of products for the elderly.

Ease of use is the focus of the current research in the field of elderly products, designers need professional design for the elderly special physical condition, can make the elderly easy to accept and use these devices, which improve the efficiency of interaction between products and users. The elderly industry is in constant development, and is expected to become a "sunrise industry" in twenty-first Century ".

At present, the elderly products in the market in a vacant State specifically for the elderly ease of use of home appliances product design has not been a good solution. And the kitchen as one of the main leisure places for the elderly after retirement, the corresponding elderly household electrical appliances design needs, is gradually enlarged. Rice cooker in the kitchen as one of the most commonly used household appliances, has long been involved in millions of households, especially in the elderly, but now the market is not on the rice pot for the elderly to consider this special population. Therefore, it is urgent to study and design the further research and design of kitchen electric cooker for the elderly.

\section{based on the design of the electric rice cooker in the elderly}

\section{based on the design goal of the electric rice cooker of the easy use of the elderly}

Ease of use of the design point of view is that the product can be used by the elderly easy and effective, and the product to achieve a variety of functions, changes in the status of the mutual link. When doing the research of this kind of function, we must first confirm the necessity and rationality of the functional connection, can not force the user's behavior needs, according to the needs of users to determine the functional requirements. After investigation and analysis, the kitchen cooker is restricted in the elderly physical function of the most frequently used products, and has a certain function in contact, interaction, so as to use the electric cooker design products of the elderly is necessary and reasonable.

the design goal of the electric rice cooker in the elderly is analyzed from the view of the ease of use.

It is advantageous to grasp the essential characteristics of product design to meet the changing characteristics of the user and realize the different functional requirements of the user's using process, from the view of ease of use. According to the design goal of ease of use aged rice cooker, specific analysis is as follows: (1) rice cookers use state in the elderly safe and easy to use; (2) adaptability, rice cookers to different objects have a certain ability to adapt; (3) effective, in the performance of electric rice with certain energy in the environment. 
the specific design of the electric rice cooker in the elderly

The design of the electric rice cooker of the easy use of the elderly should be based on the group itself, considering the physiological characteristics and psychological characteristics of the elderly. This easy to use electric rice cooker mainly for the analysis of the above analysis, in order to adapt to the characteristics of the elderly's body function as the breakthrough point, in the design of the knot intelligent, material, technology and aesthetics are closely linked.

(1) appearance. The design of the elderly electric rice cooker modeling fashion lightweight, more use of mild curve based, in the corner of the line using a large arc $\mathrm{R}$ angle processing, so that the product has a sense of affinity, easy to be accepted by the elderly. The operation of electric rice cooker is convenient and safe.

(2) function. Elderly rice cooker with the basic functions, while in order to facilitate the operation of the elderly, the use of intelligent design, guide the elderly to use this product easy to understand: operating tips, such as lighting, voice prompts, etc..

(3) color. The design of the elderly kitchen appliances, in color, to cater to the elderly more visual experience, from the color hue, lightness and purity of. For older people to create a comfortable and carefree feeling. Therefore, the color of the elderly electric rice cooker mainly in the classic black, white, low saturation of gray based, and a single color, to reveal the simple, natural atmosphere, but also with the kitchen environment. The use of bright colors in the function area, to prompt, warning.

(4) material. Different materials, to give people different feelings, now the old product material is mainly plastic, wood, ceramics, metal alloys, plastics, which used more, wood, ceramics used in special products. Taking into account the user for the elderly, select the material to be non slip.

\section{Conclusions}

Now, China's aging phenomenon is more serious, many elderly health products are widely popular, however, in the market on the use of household electrical appliances products are still in the embryonic stage. The elderly are vulnerable groups in society, is an important part of China population, regardless of the elderly health status of this group is starting from the product benefits, ease of use are the focus of product design in old people, a simple use of security products can not only give the elderly, the elderly life the convenience, at the same time can bring considerable economic benefits for manufacturers.

\section{Acknowledgements}

This work was financially supported by the Guangxi Middle-aged and young teachers' basic ability promotion project of 2016(ky2016YB139).

\section{References}

[1] Zhang Lixia. Analysis of consumer psychology and consumer behavior of the elderly. contemporary economy, Vol. 11:32-33(2013),.

[2] Yu Tao. Study on the aging of China's population and the consumption of the aged. Jilin University( 2013).

[3] Liu Liu, Yang Yu, Sun Tao. The design of the advanced aged product based on human computer interaction. industrial engineering, Vol. 05:89-94(2010).

[4] Wang Dexiang. China's demand for home appliances secret. popular science, Vol.12:32 (2013).

[5] Miao Rui. The design of the ease of use of home appliances for the elderly. Qiqihar University (2013) . 
[6] Yang $\mathrm{Zu}$. The man-machine interface design of the home appliance used by the aged, Beijing University of science and Technology Press (2010). 